\title{
SN 1999ga: a low-luminosity linear type II supernova?
}

\author{
A. Pastorello ${ }^{1}$, R. M. Crockett ${ }^{1}$, R. Martin ${ }^{2}$, S. J. Smartt ${ }^{1}$, G. Altavilla ${ }^{3}$, S. Benetti ${ }^{4}$, M. T. Botticella ${ }^{1}$, E. Cappellaro ${ }^{4}$,

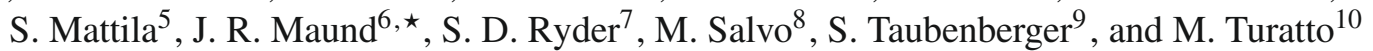

\author{
1 Astrophysics Research Centre, School of Mathematics and Physics, Queen's University Belfast, Belfast BT7 1NN, UK \\ e-mail: a.pastorello@qub.ac.uk \\ 2 Perth Observatory, 337 Walnut Road, Bickley 6076, Perth, Australia \\ 3 INAF - Osservatorio Astronomico di Bologna, via Ranzani 1, 40127 Bologna, Italy \\ ${ }^{4}$ INAF - Osservatorio Astronomico di Padova, Vicolo dell' Osservatorio 5, 35122 Padova, Italy \\ 5 Tuorla Observatory, Department of Physics \& Astronomy, University of Turku, Väisäläntie 20, 21500 Piikkiö, Finland \\ 6 Dark Cosmology Centre, Niels Bohr Institute, University of Copenhagen, Julian Maries Vej 30, 2100 Copenhagen, Denmark \\ 7 Anglo-Australian Observatory, PO Box 296, Epping, NSW 1710, Australia \\ 8 Research School of Astronomy and Astrophysics, Australian National University, Mount Stromlo and Siding Spring Observatories \\ Cotter Road, Weston Creek, ACT 2611, Australia \\ 9 Max-Planck-Institut für Astrophysik, Karl-Schwarzschild-Str. 1, 85741 Garching bei München, Germany \\ 10 INAF - Osservatorio Astrofisico di Catania, via S. Sofia 78, 95123 Catania, Italy
}

Received 6 March 2009 / Accepted 28 March 2009

\section{ABSTRACT}

\begin{abstract}
Context. Type II-linear supernovae are thought to arise from progenitors that have lost most of their $\mathrm{H}$ envelope by the time of the explosion, and they are poorly understood because they are only occasionally discovered. It is possible that they are intrinsically rare, but selection effects due to their rapid luminosity evolution may also play an important role in limiting the number of detections. In this context, the discovery of a subluminous type II-linear event is even more interesting.

Aims. We investigate the physical properties and characterise the explosion site of the type II SN 1999ga, which exploded in the nearby spiral galaxy NGC 2442.

Methods. Spectroscopic and photometric observations of SN 1999ga allow us to constrain the energetics of the explosion and to estimate the mass of the ejected material, shedding light on the nature of the progenitor star in the final stages of its life. The study of the environment in the vicinity of the explosion site provides information on a possible relation between these unusual supernovae and the properties of the galaxies hosting them.

Results. Despite the lack of early-time observations, we provide reasonable evidence that SN 1999ga was probably a type II-linear supernova that ejected a few solar masses of material, with a very small amount of radioactive elements of the order of $0.01 M_{\odot}$.
\end{abstract}

Key words. stars: supernovae: general - stars: supernovae: individual: SN 1999ga - stars: supernovae: individual: SN 1979C stars: supernovae: individual: SN 1980K - stars: supernovae: individual: SN 1990K

\section{Introduction}

Type II-linear supernovae (SNe IIL) form a rare and poorly studied class of core-collapse supernovae ( $\mathrm{SNe})$. Contrary to type II-plateau supernovae (SNe IIP) that show slow photometric evolution and well-correlated observed properties (see e.g. Hamuy 2003, and references therein), SNe IIL are characterised by fast-evolving light curves and a higher degree of heterogeneity. SNe IIL are believed to arise either from the explosion of moderate-mass progenitors $\left(8-10 M_{\odot}\right)$ that have lost a significant fraction of their $\mathrm{H}$ envelope via binary interaction or from more massive stars that lose mass before their explosion through strong stellar winds.

However, despite the mass loss, a significant amount of $\mathrm{H}$ still remains in the stellar envelope, and, consequently, the spectrum of a SN IIL still shows prominent $\mathrm{H}$ lines. The evidence that in SNe IIL the H envelope is not as massive as in SNe IIP comes mostly from analysis of the light curves. Because of the lack of a massive $\mathrm{H}$ envelope that recombines with cooling, the light curves do not show the characteristic long period (about

\footnotetext{
* Sopie and Tycho Brahe Fellow.
}

three months) of almost constant luminosity typical of SNe IIP, but instead show a linear decline after maximum.

In Patat et al. $(1993,1994)$ a compilation of light curves from the literature of a large number of SNe II was analysed. A high degree of heterogeneity in the light curve shapes was found, and many transitional objects between type IIP and IIL SNe were shown to exist. This is probably a consequence of the progressively declining mass of the residual $\mathrm{H}$ envelope along the sequence from type IIP to type IIL events. Up to the point of explosion, the progenitors of type IIP SNe have retained much of their H-rich envelopes. On the other hand, stars generating SNe IIL have already lost most of their $\mathrm{H}$ envelopes at the time of the explosion. In a few cases, the presence of this circumstellar material (CSM) is revealed at some point because of the interaction with the expanding ejecta. However, in general, this is not the dominant contribution to the luminosity of SNe IIL, at least at early phases. A consequence of this is that SNe IIL show a much more rapid photometric evolution than type IIn $\mathrm{SNe}$ (Schlegel 1990), in which the ejecta-CSM interaction is the main process regulating the output of energy of the $\mathrm{SN}$ even at early phases. Nevertheless, a few type II SNe show unequivocal evidence of interaction with CSM from specific spectral 
properties and the high peak luminosity without showing slowly evolving light curves. This is the case for a few SNe IIn with fastdeclining light curves, like SN 1998S (e.g. Fassia et al. 2000; Liu et al. 2000), SN 1999el (Di Carlo et al. 2002), or SN 2006jc (and SNe Ibn) which interact with a He-rich CSM (Pastorello et al. 2007; Foley et al. 2007; Pastorello et al. 2008a). All these $\mathrm{SNe}$ have light curves with rapid evolution resembling those of SNe IIL.

The prototype of the classical SNe IIL is SN 1979C, which exploded in M100 and is by far the best studied object of this class, being extensively observed from X-ray to radio wavelengths (see e.g. Panagia et al. 1980; de Vaucouleurs et al. 1981; Branch et al. 1981; Weiler et al. 1991; Fesen \& Matonick 1993; Immler et al. 1998; Fesen et al. 1999; van Dyk et al. 1999; Montes et al. 2000; Ray et al. 2001; Marcaide et al. 2002; Bartel \& Bietenholz 2003; Immler et al. 2005). Other well studied SNe IIL discovered in the last 30 years are SN 1980K in NGC 6946 (see e.g. Buta 1982; Barbon et al. 1982a for earlytime data; and Uomoto \& Kishner 1986; Fesen et al. 1999, and references therein, for late-time data), SN 1986E in NGC 4302 (Cappellaro et al. 1990, 1995a) and SN 1990K in NGC 150 (Cappellaro et al. 1995b).

A few SNe IIL, sometimes dubbed as type IId SNe (Benetti 2000), show narrow P-Cygni Balmer lines of hydrogen superimposed on otherwise normal broad-lined spectra of type II SNe. This is interpreted as an evidence of super-winds occurred years to decades before the SN explosions. This group includes SNe 1994aj, 1996L, 1996al, 2000P (Benetti et al. 1998, 1999; Benetti \& Neuhauser 1996; Benetti 2000; Cappellaro et al. 2000; Jha et al. 2000). In all these cases, ejecta-CSM interaction takes place a few years after the explosion, when the fast SN ejecta reaches the slower wind. The ejecta-CSM interaction may be heralded by a number of different pieces of observational evidence, e.g. flattening of the late-time optical light curves, the peculiar profile of spectral lines (e.g. Schlegel 1990; Turatto et al. 1993a; Leibundgut et al. 1991; Fesen et al. 1999), strong X-ray (see Schlegel 1995; Immler et al. 1998; Aschenbach 2007, for reviews) and radio emission (e.g. van Dyk et al. 1996).

A special mention has to be given to the recently discovered type IIL SNe that have extremely high luminosities $\left(M_{V}<\right.$ -22.5). This small sub-group includes SN 2005ap (Quimby et al. 2007) and SN 2008es (Miller et al. 2009; Gezari et al. 2009). Their extraordinary luminosity and their spectra, which show no evidence of the narrow features typical of interacting objects (SNe IIn), are best explained by strong interaction of the ejecta with a dense, optically-thick CSM, rather than with very large ejected ${ }^{56} \mathrm{Ni}$ masses (Quimby et al. 2007).

In this paper we study the case of SN 1999ga, a type II SN (likely of type IIL) which was followed only at late phases. Some pre-explosion, ground-based images, and very late time Hubble Space Telescope (HST) frames have been studied in order to constrain the characteristics of the progenitor star and the site of explosion. Basic information on SN 1999ga and its spectacular host galaxy is reported in Sect. 2. Photometric and spectroscopic data are presented in Sects. 3 and 4, respectively. The explosion site, imaged in pre-SN archival observations and in deep HST frames obtained years after the SN explosion, is analysed in Sect. 5, while a discussion and a summary follow in Sect. 6.

\section{SN 1999ga in NGC 2442}

SN 1999ga was discovered on November 19.76 UT in the spiral galaxy NGC 2442 by Woodings et al. (1999), on behalf of the Perth Astronomy Research Group (PARG, see e.g.
Woodings et al. 1998), in the course of an automated supernova search performed with the Perth-Lowell 0.61-m reflector. The discovery magnitude quoted by Woodings et al. (1999), $R \sim 18$, is wrong, being almost 2 mag fainter than the true magnitude of SN 1999ga at that epoch (cf. Sect. 3). The exact position was measured on Nov. 22.54 with the 0.25-m Mike Candy Telescope at Perth Observatory and found to be RA $=7^{\mathrm{h}} 36^{\mathrm{m}} 16^{\mathrm{s}} .70 \pm 0.03$, Dec $=-69^{\circ} 33^{\prime} 21^{\prime \prime} .8 \pm 00^{\prime} .4$ (equinox 2000.0), about $38^{\prime \prime}$ west and 91" south of the centre of NGC 2442 (Woodings et al. 1999).

Additional observations, obtained on November 25.18 UT by Rubinstein (1999) at the Cerro Tololo Interamerican Observatory, indicated that the magnitude of the transient was significantly brighter than that reported by Woodings et al. (1999) $(B=18.3 \pm 0.3, V=17.1 \pm 0.1, R=16.2 \pm$ $0.1, V=15.6 \pm 0.1)$. On the basis of the SN location alone, Rubinstein (1999) suggested a possible type II classification for SN 1999ga. Because of its red colour, he also suggested significant interstellar reddening. However, we will see in Sects. 3 and 4 that SN 1999ga was discovered late, and it is well known that type II SNe are intrinsically red at the end of the photospheric phase (e.g. Pastorello et al. 2004).

The tentative SN classification of Rubinstein (1999) was confirmed spectroscopically by Salvo et al. (1999) on the basis of a spectrum taken on December 29 at ESO-La Silla. The classification spectrum (presented in this paper) shows that SN 1999ga is a type II SN observed a few months after explosion. In this paper we will adopt JD $=2451420$ as an indicative epoch for the explosion of SN 1999ga, which was derived via comparisons with observed data of well-studied type II SNe (Sects. 3 and 4).

The spectral lines in the classification spectrum show P-Cygni profiles, while $\mathrm{H} \alpha$ has a peculiar flat-topped emission component indicative that the $\mathrm{H} \alpha$ emission mostly comes from a shell-like region (see Sect. 4 for discussion). Finally the spectrum shows evidence of little interstellar extinction (Salvo et al. 1999), suggesting moderate reddening.

The spectacular galaxy hosting SN 1999ga, NGC 2442 (Fig. 1), is classified by HyperLeda ${ }^{1}$ as an SBbc galaxy, with well-developed but asymmetric spiral arms. It belong to the Volans group of galaxies, and Ryder et al. (2001) report a distance of about $15.5 \mathrm{Mpc}$ (distance modulus $\mu=30.95 \mathrm{mag}$ ). From the recessional velocity corrected by Local Group infall into Virgo, $1150 \mathrm{~km} \mathrm{~s}^{-1}$, and assuming $H_{0}=72 \mathrm{~km} \mathrm{~s}^{-1} \mathrm{Mpc}^{-1}$, we obtain a slightly higher distance, $d \approx 16 \mathrm{Mpc}(\mu=$ $31.02 \mathrm{mag}$ ), which will be adopted throughout this paper. The Galaxy extinction in the direction of NGC 2442 is rather high, being $E(B-V)_{\mathrm{Gal}}=0.20 \mathrm{mag}$ (Schlegel et al. 1998). The low signal to noise and the late phase of the SN 1999ga spectra (see Sect. 4) do not allow precise measurement of the equivalent width $(E W)$ of Galaxy and host galaxy interstellar Na I doublet (Na ID) absorptions. However, we tentatively estimated the two components of $\mathrm{Na}$ ID in the SN spectra and found that the NGC 2442 component has an $E W \approx 0.9 \AA$, which is roughly 70 per cent that of the Galaxy. This would imply an $E(B-V)_{\text {host }}=$ $0.14 \mathrm{mag}$, which is consistent with what we find using the correlation between $E W$ of the interstellar Na ID and $E(B-V)$ of Turatto et al. (2003). Accounting for both the Galactic reddening component and that of the host galaxy, we obtain $E(B-V)_{\text {tot }}=$ 0.34 mag as our best estimate for the total reddening, and this value will be adopted hereafter.

Several attempts have been made in order to explain the disturbed morphology of NGC 2442, by searching for evidence of tidal interaction with a few nearby galaxies

${ }^{1}$ http://leda.univ-lyon $1 . f r /$ 


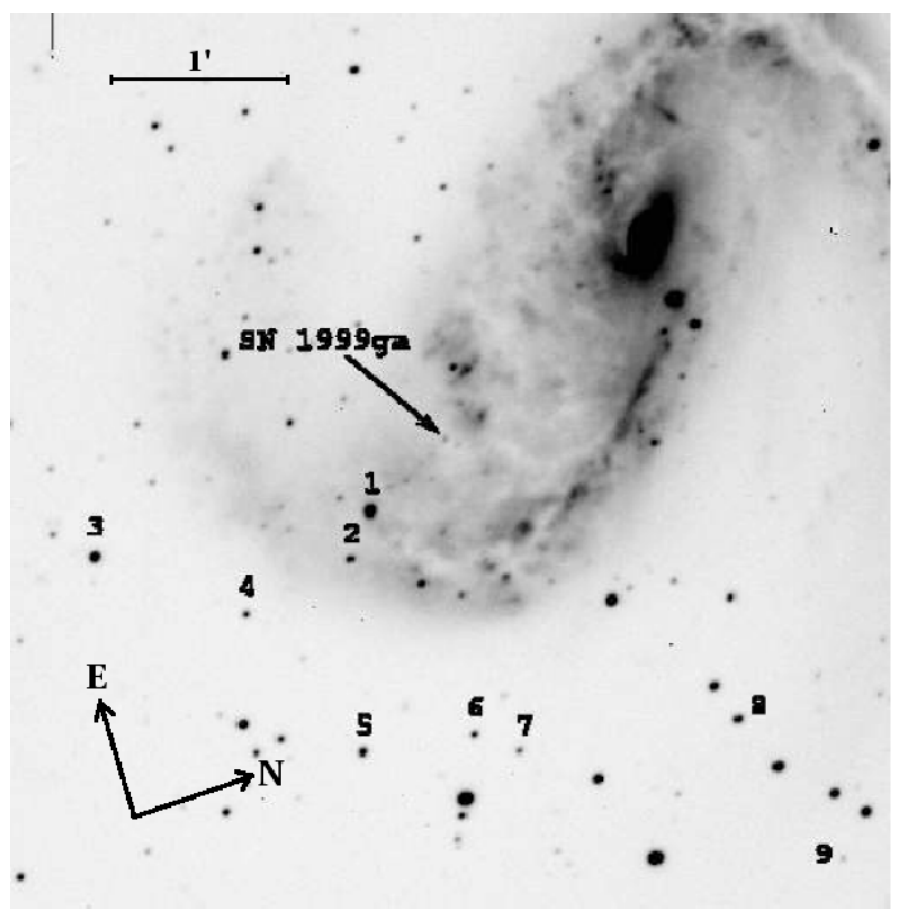

Fig. 1. SN 1999ga and NGC 2442. R-band image obtained on Apr. 7, 2000 with the ESO $3.6 \mathrm{~m}$ Telescope equipped with EFOSC2. A sequence of reference stars is marked by numbers.

(Elmegreen et al. 1991; Sandage \& Bedke 1994; Mihos \& Bothun 1997; Houghton 1998). Ryder et al. (2001) found evidence that NGC 2442 is associated with an extremely massive cloud of H I, with a mass almost one third that of the galaxy itself. This gas cloud was probably produced in a recent tidal encounter with a moderately massive companion, though both ram pressure-stripping, and HI rings/arcs stripped from the outer envelope of a low surface brightness galaxy (Bekki et al. 2005) are also possible scenarios. Moreover, Bajaja et al. (1995), Mihos \& Bothun (1997) and Bajaja et al. (1999) found an elliptical, circumnuclear, star-forming molecular ring. Finally, the intensity ratios of emission lines of the galaxy nucleus indicate that NGC 2442 is likely a LINER (Bajaja et al. 1999).

\section{Light curve}

Follow-up photometric observations were carried out using a number of telescopes in Australia and Chile. Available imaging of SN 1999ga was reduced following a standard procedure (see e.g. Pastorello et al. 2005): the images were first overscan, bias and flat-field corrected. SN magnitudes were then measured using a PSF-fitting technique after the subtraction of template images. The recovered magnitudes were then scaled using the night zero-points computed comparing the instrumental magnitudes of several well-known standards fields with those reported in the Landolt catalogue (Landolt 1992). Finally the SN magnitudes were fine tuned with reference to the magnitudes of 9 stars in the field of NGC 2442 (see Fig. 1) obtained by averaging the estimates obtained in photometric nights. The magnitudes of the sequence stars, as denoted in Fig. 1, are reported in Table 1, while the $B, V, R$ and $I$ magnitudes of SN 1999ga are given in Table 2.

In Fig. 2 the photometric evolution of SN 1999ga in the optical bands is shown. The late discovery of SN 1999ga prevents us from studying the early evolution of its light curve, with
Table 1. Magnitudes of reference stars in the SN field. The rms of the average magnitudes are reported in brackets.

\begin{tabular}{ccccc}
\hline \hline Star & $B$ & $V$ & $R$ & $I$ \\
\hline 1 & $17.55(0.02)$ & $16.55(0.01)$ & $15.89(0.02)$ & $15.18(0.02)$ \\
2 & $20.02(0.02)$ & $18.48(0.01)$ & $17.52(0.01)$ & $16.37(0.02)$ \\
3 & $18.29(0.03)$ & $16.84(0.01)$ & $15.92(0.05)$ & $15.05(0.03)$ \\
4 & $19.45(0.02)$ & $18.73(0.01)$ & $18.23(0.01)$ & $17.73(0.02)$ \\
5 & $18.82(0.01)$ & $18.05(0.01)$ & $17.59(0.01)$ & $17.09(0.01)$ \\
6 & $20.33(0.02)$ & $18.76(0.01)$ & $17.77(0.01)$ & $16.71(0.02)$ \\
7 & $20.03(0.01)$ & $19.15(0.02)$ & $18.57(0.01)$ & $18.08(0.02)$ \\
8 & $19.41(0.04)$ & $18.21(0.03)$ & $17.38(0.04)$ & $16.80(0.03)$ \\
9 & & $20.36(0.04)$ & & $18.05(0.02)$ \\
\hline
\end{tabular}

particular reference to the immediate post-maximum behaviour. As a consequence, uncertainty as to the shape of its light curve (linear or plateau) remains, although there is some evidence in favour of a type IIL classification for SN 1999ga (see Sect. 4).

SN 1999ga was discovered during the post-maximum decline phase. The light curve shows a rapid luminosity drop (by 1.5-2 mag in all bands), followed by a less steep decline corresponding to the exponential (radioactive) tail. As evidenced in Fig. 2, at late time the $V$-band light curve of SN 1999ga closely matches the ${ }^{56} \mathrm{Co} \rightarrow{ }^{56} \mathrm{Fe}$ decay slope $\left(0.98 \mathrm{mag} / 100^{d}\right.$, assuming complete gamma-ray trapping), even though at very late phases (from $\sim 400$ days after explosion) the light curves in all bands show steeper declines. This is has been seen in other type II SNe, and it is generally interpreted as dust forming in the SN ejecta and/or incomplete $\gamma$-ray trapping (e.g. Elmhamdi et al. 2003).

The slopes in the period between 120 and 450 days, as derived from a least squares fit to the light curves, are $\gamma_{B}=$ $0.87 \mathrm{mag} / 100^{d}, \gamma_{V}=1.02 \mathrm{mag} / 100^{d}, \gamma_{R}=1.15 \mathrm{mag} / 100^{d}$ and $\gamma_{I}=1.00 \mathrm{mag} / 100^{d}$, on average rather consistent with those expected by the radioactive decay of ${ }^{56} \mathrm{Co}$ in the case of complete $\gamma$-ray trapping. This might be an indication of relatively massive ejecta.

In Fig. 3 (top panel) the $V$-band absolute light curve of SN 1999ga is compared with those of a number of type II SNe, four SNe IIL (1979C, 1980K, 1990K and the peculiar 1994aj, which belongs to the IId sub-type, Benetti 2000), the overluminous type IIL SN 2008es, the transitional type IIP/IIL SN 1992H and three SNe IIP (2004et, 2005cs and the peculiar 1987A). The phases for SN 1999ga have been computed assuming JD $=2451420$ as an indicative epoch for the corecollapse, as derived from a comparison of the light curve and spectra with those of other SNe II. The apparent flattening in the very early light curve of SN 1999ga would suggest a plateaulike behaviour (or, at least, a transitional object). However, Patat et al. $(1993,1994)$ noted that a shoulder in the light curves of SNe IIL (especially in the $R$ band) is frequently observed after maximum. Hence the available photometry of SN 1999ga does not allow us to definitely discriminate between a linear or a plateau type light curve. We will see in Sect. 4 that more clear clues in favour of a type IIL classification for SN 1999ga come from the spectroscopy.

With the assumption on the explosion epoch mentioned above, a comparison between the integrated late-time BVRI luminosity of SN 1999ga with that of SN 1987A at similar epochs provides an approximate estimate of the ${ }^{56} \mathrm{Ni}$ mass synthesized by SN 1999ga, being about $0.013 \pm 0.003 M_{\odot}$, which is to our knowledge the smallest ever registered for a type IIL SN, and only marginally higher than those registered for the lowluminosity SNe IIP (e.g. Pastorello et al. 2004). 
Table 2. Calibrated magnitudes of SN 1999ga. An additional detection limit in the $U$ band $(U>21.9)$ was obtained on 1999 , December 29.

\begin{tabular}{|c|c|c|c|c|c|c|}
\hline$\overline{\text { Date }}$ & $\overline{\mathrm{JD}}$ & $\overline{\bar{B}}$ & $\overline{\bar{V}}$ & $\overline{\bar{R}}$ & $I$ & Instrument \\
\hline 1999-11-19 & 2451502.29 & & $16.90(0.08)$ & $16.11(0.05)$ & $15.40(0.04)$ & PARG \\
\hline 1999-11-24 & 2451507.29 & & $17.00(0.07)$ & $16.18(0.06)$ & $15.46(0.06)$ & PARG \\
\hline 1999-11-26 & 2451509.02 & & $16.98(0.04)$ & $16.18(0.03)$ & $15.45(0.04)$ & PARG \\
\hline 1999-12-02 & 2451514.84 & & & $16.30(0.03)$ & $15.58(0.05)$ & PARG \\
\hline $1999-12-10$ & 2451522.79 & & $17.36(0.25)$ & $16.46(0.04)$ & $15.70(0.14)$ & PARG \\
\hline $1999-12-13$ & 2451525.84 & & $17.53(0.05)$ & $16.62(0.04)$ & $15.86(0.04)$ & PARG \\
\hline $1999-12-20$ & 2451532.87 & & $17.86(0.07)$ & $17.05(0.05)$ & $16.28(0.07)$ & PARG \\
\hline $1999-12-26$ & 2451538.82 & $20.45(0.07)$ & & & & $\mathrm{WFI}^{\star}$ \\
\hline 1999-12-29 & 2451541.77 & $21.03(0.08)$ & $19.13(0.03)$ & $18.05(0.04)$ & $17.36(0.06)$ & DF \\
\hline 2000-01-01 & 2451546.27 & & $19.19(0.42)$ & $18.11(0.21)$ & $17.40(0.32)$ & PARG $^{\circ}$ \\
\hline 2000-01-29 & 2451573.13 & $21.29(0.30)$ & $19.57(0.04)$ & $18.35(0.07)$ & & $\mathrm{AAT}^{*}$ \\
\hline 2000-02-17 & 2451591.67 & $21.46(0.13)$ & $19.74(0.07)$ & $18.42(0.03)$ & & $\mathrm{WFI}^{\star}$ \\
\hline $2000-03-13$ & 2451617.02 & & $20.0(0.3)$ & $18.8(0.3)$ & $18.4(0.4)$ & $\mathrm{DF}^{\dagger}$ \\
\hline 2000-03-14 & 2451617.5 & & $19.98(0.07)$ & & & DF \\
\hline 2000-04-07 & 2451641.57 & $21.89(0.05)$ & $20.26(0.03)$ & $19.01(0.07)$ & & EF2 \\
\hline 2000-11-19 & 2451867.85 & & $22.55(0.24)$ & $21.79(0.21)$ & $20.69(0.24)$ & DF \\
\hline 2001-02-02 & 2451942.75 & $>23.1$ & $23.66(0.38)$ & $23.02(0.47)$ & & EF2 \\
\hline 2001-02-04 & 2451944.63 & & $>23.3$ & $>22.5$ & & WFI \\
\hline 2001-03-17 & 2451985.61 & $>23.6$ & $>22.9$ & $>22.4$ & $>23.7$ & DF \\
\hline
\end{tabular}

* Anglo Australian Telescope Data Archive: http://archive.ast.cam.ac.uk/arc-bin/wdb/aat_database/observation_log/make; * ESO Data Archive: http://archive.eso.org/eso/eso_archive_main.html; ${ }^{\circ}$ poor night; ${ }^{\dagger}$ spectrophotometric magnitude. PARG = Perth-Lowell 0.61-m Cassegrain Telescope + CCD (Perth, Australia); EF2 = ESO 3.6 m Telescope + EFOSC2 (La Silla, Chile); WFI = ESO/MPI 2.2 m Telescope + Wide Field Imager (La Silla, Chile); DF = Danish 1.54 m Telescope + DFOSC (La Silla, Chile).

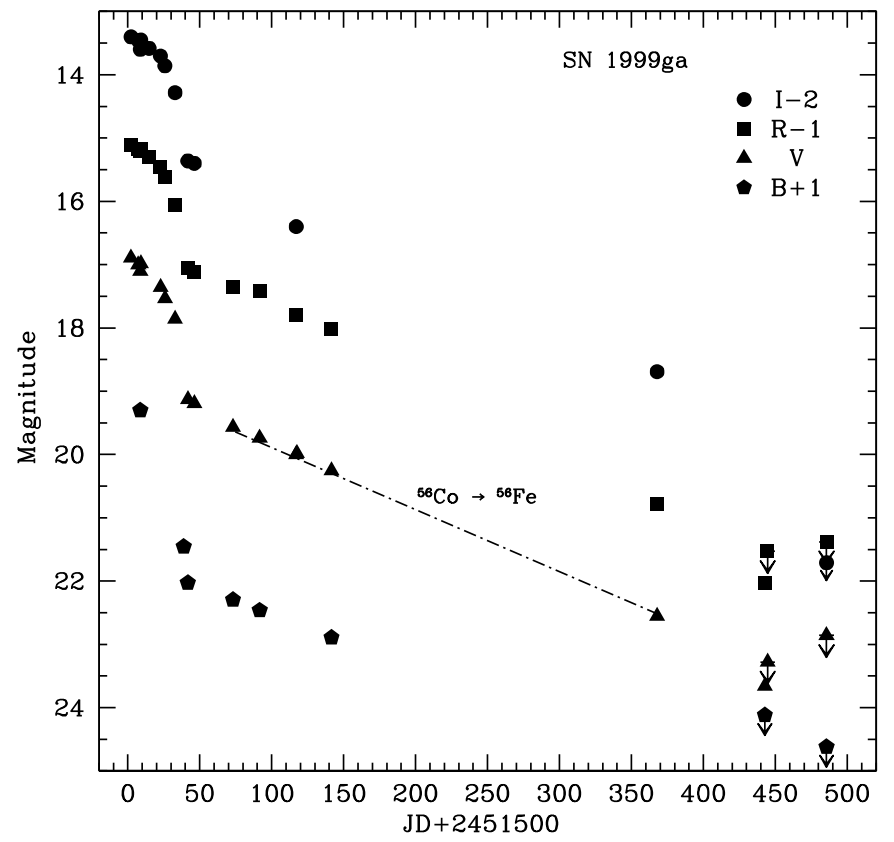

Fig. 2. $B, V, R, I$ light curves of SN 1999ga. Unfortunately, only late time photometry is available for this $\mathrm{SN}$, because the $\mathrm{SN}$ was not observed at early epochs as the host galaxy was too low on the horizon for most of the night. Another seasonal gap is visible between JD = 2451650 and JD $=2451850$. The photometric points from Rubenstein (1999) are also included.

A comparison among $B-V$ colour curves of SN 1999ga and some other SNe II is also shown in Fig. 3 (bottom panel). Surprisingly, the overall behaviour of the colour curve of the type IIL SN 1990K within the first 250 days closely resembles that of SN 1987A, more than that of other SNe IIL shown in figure. However, we should remember that the luminous light curve peaks and the bluer colours of SN 1979C and SN 1980K are probably signatures of interaction with a CSM (see Sect. 6).
Unfortunately, only a few points are available for SN 1999ga and this does not allow us to study in detail its colour evolution. At $\sim 120$ days, the $B-V$ colour of $1999 \mathrm{ga}$ is around $1.5 \mathrm{mag}$, then it becomes marginally bluer (by about $1.3 \mathrm{mag}$ ) 4 months later. However, the overall similarity with the colour evolutions of SNe 1987A, 2004et, 1992H and 1990K (between +110 and +230 days) is an additional argument in favour of a late discovery of SN 1999ga.

\section{Late spectral evolution}

Four spectra of SN 1999ga have been obtained with the ESO telescopes at La Silla (Chile), and basic information about these spectra is reported in Table 3. The available spectral sequence is shown in Fig. 4.

The earliest spectrum is typical for a type II SN transiting from the photospheric to the nebular phase. Prominent $\mathrm{H} \alpha$ and $\mathrm{H} \beta$ are visible, with relatively weak P-Cygni absorptions, along with strong P-Cygni lines of Na ID and O I $\lambda 7774$. The minima of the absorption components of $\mathrm{H} \alpha$ and $\mathrm{Na}$ ID are blueshifted by about $5800 \mathrm{~km} \mathrm{~s}^{-1}$ and $5300 \mathrm{~km} \mathrm{~s}^{-1}$, respectively. The feature at about $8600 \AA$ is attributed to a blend of O I $\lambda 8446$ plus the Ca II near-infrared triplet. A few Fe II lines are possibly detected near the region of $\mathrm{H} \beta$. It is worth to note that $\mathrm{H} \alpha$ shows an unusual flat-topped emission profile. A photospheric spectrum showing a $\mathrm{P}-\mathrm{Cygni} \mathrm{H} \alpha$ line with flat-topped profile is indicative that the $\mathrm{H} \alpha$ emission is mostly produced in a detached region. Detached atmospheres are not common in SNe (Jeffery \& Branch 1990) and this is the first time we see such a structure in a type II supernova. This could be caused by a temporary peak in the density profile of the hydrogen layer. A flat-topped profile may be also produced in a cool dense shell (CDS) that forms at the interface between the SN ejecta and the wind produced by the progenitor (Chugai et al. 2007).

The evolution of SN 1999ga is slow and the two subsequent spectra show basically the same features as the first spectrum, plus much stronger nebular lines of [O I] $\lambda \lambda 6300-6364$ 
Table 3. Spectroscopic observations of SN 1999ga. The spectrum at 39.5 days is the one used to classify SN 1999ga (Salvo et al. 1999).

\begin{tabular}{ccccccc}
\hline \hline Date & JD $(+2400000)$ & Phase $^{\dagger}$ & Instrumental configuration & Exposure & Resolution $(\AA)$ & Range $(\AA)$ \\
\hline $1999-12-29$ & 51541.76 & 39.5 & Danish 1.54 m + DFOSC + gr.4 & $1200 \mathrm{~s}$ & 11 & $3650-9050$ \\
$2000-03-12 / 13$ & 51617.02 & 114.8 & Danish 1.54 m + DFOSC + gr.4 + gr.5 & $1800 \mathrm{~s}+1800 \mathrm{~s}$ & $11+12$ & $3570-10200$ \\
$2000-04-07$ & 51641.58 & 139.3 & ESO 3.6 m + EFOSC2 + gr.11 & $2 \times 1800 \mathrm{~s}$ & 18 & $3380-7500$ \\
$2001-02-02$ & 51942.71 & 440.5 & ESO 3.6 m + EFOSC2 + gr.11 & $3 \times 1800 \mathrm{~s}$ & 18 & $3490-7460$ \\
\hline
\end{tabular}

$\dagger$ Days from discovery (JD = 2451502.26$)$; ${ }^{\ddagger}$ spectrum obtained averaging two spectra obtained in subsequent days.

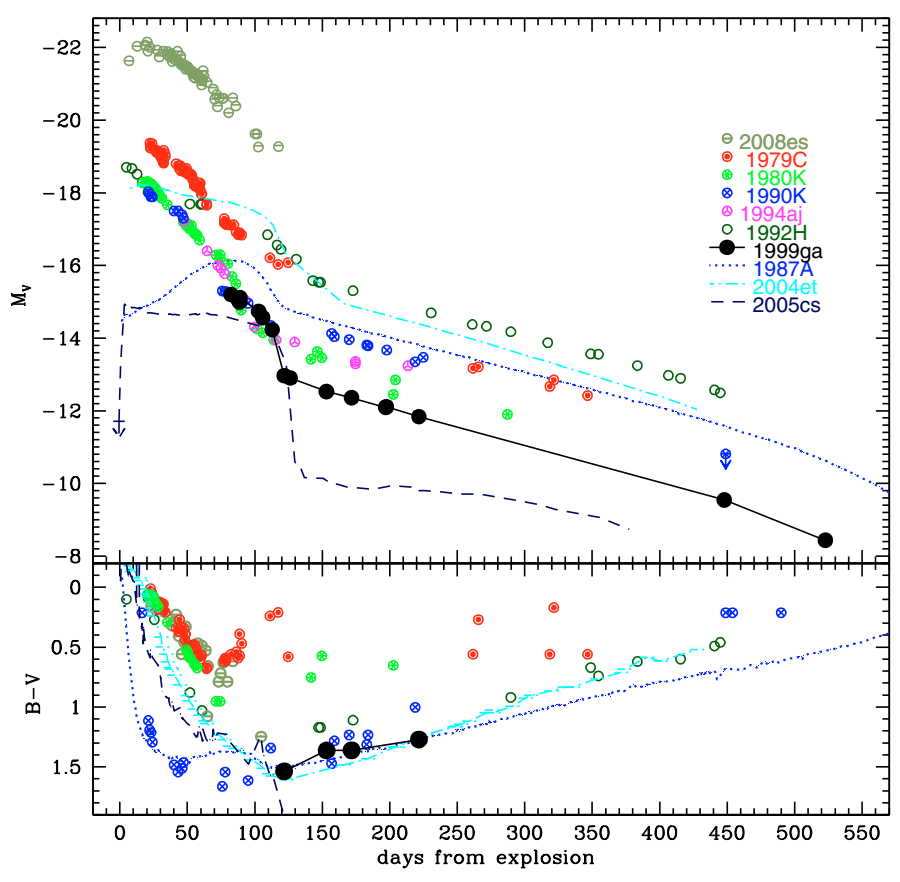

Fig. 3. Top: $V$-band absolute light curves of SN 1999ga and a number of type II SNe: the high-luminosity SN 2008es (Gezari et al. 2009; Miller et al. 2009), the type IIL SNe 1979C (Balinskaya et al. 1980; de Vaucouleurs et al. 1981; Barbon et al. 1982b), 1980K (Barbon et al. 1982a; Buta 1982; Tsvetkov 1983), 1990K (Cappellaro et al. 1995b), 1994aj (Benetti et al. 1998); the transitional type IIP/IIL SN 1992H (Clocchiatti et al. 1996); the peculiar SN 1987A (Whitelock et al. 1989, and references therein) and the type IIP SNe 2004et (Sahu et al. 2006; Misra et al. 2007) and 2005cs (Tsvetkov et al. 2006; Pastorello et al. 2006, 2009). The explosion epochs of SNe IIL have been estimated to occur roughly 2-3 weeks before their maximum light (Patat et al. 1993). Bottom: comparison of the $B-V$ colour curves of SNe 1999ga, 2008es, 1979C, 1980K, 1990K, 1992H, 1987A, 2004et.

and the classical feature around $7300 \AA$ due to a blend of [Ca II] $\lambda \lambda 7291,7324,[\mathrm{O}$ II] $\lambda \lambda 7320,7230$ and [Fe II]. The $\mathrm{H} \alpha$ feature is narrower and, in contrast to the December 29 spectrum, has evolved developing a rounded profile. In the ejectawind interaction scenario of Chugai et al. (2007), this evolution of $\mathrm{H} \alpha$ is expected to be accompained by an absorption component that progessively shifts toward redder wavelengths. This is not observed in SN 1999ga, making the CDS scenario quite unlikely.

Interestingly, the two intermediate spectra of SN 1999ga show $\mathrm{H} \alpha$ with a slightly blue-shifted peak (see Fig. 4). Blueshifted emission peaks are observed in many young type II SNe (see e.g. Turatto et al. 1993b) and this in agreement with theoretical predictions (Jeffery \& Branch 1990; see also Dessart $\&$ Hillier 2008), but unexpected at late phases, unless caused by the formation of dust in the SN ejecta (like in SN 1999em, Elmhamdi et al. 2003). However the dust formation in the

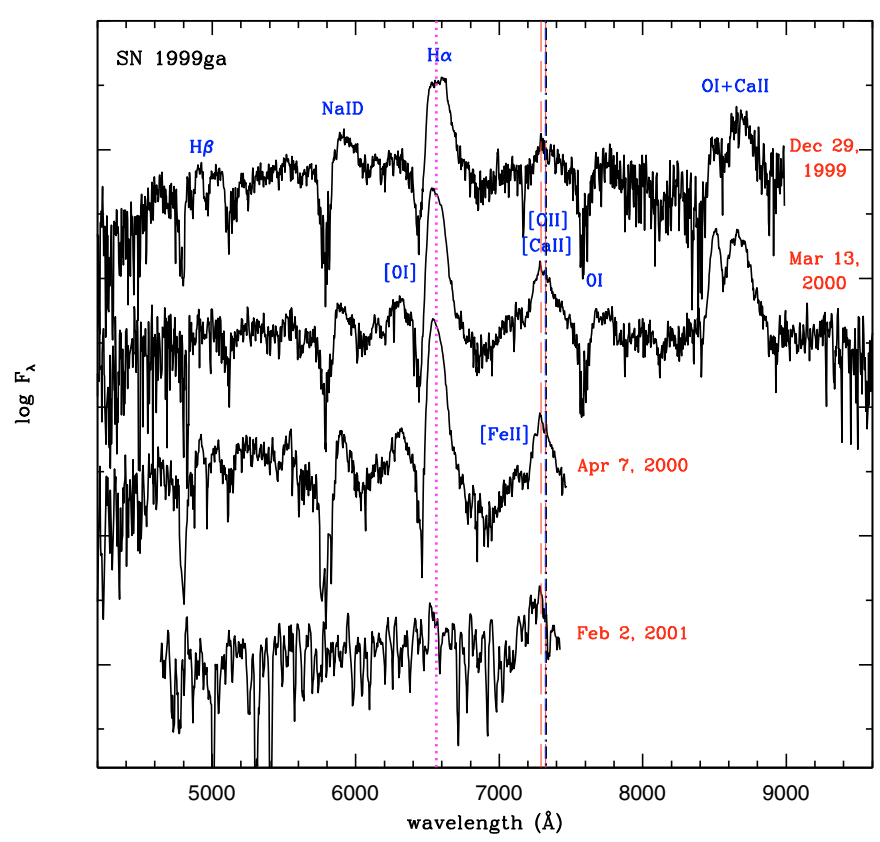

Fig. 4. Sequence of late-time spectra of SN 1999ga. The main features are labelled. The vertical lines mark the rest positions of $\mathrm{H} \alpha$ (magenta, dotted line), [Ca II] $\lambda \lambda 7291,7324$ (red, dashed lines) and [O II] $\lambda \lambda 7320$, 7330 (blue, dot-dashed lines). There is minor evidence of residual $\mathrm{H} \alpha$ in the February 2, 2001 spectrum. All spectra, reported at the host galaxy rest wavelength, have been reddening corrected.

SN ejecta is usually accompanied by an increased slope of the optical light curves and, eventually, an IR excess. Interestingly, in the case of SN 1999ga a deviation from the ${ }^{56}$ Co decay slope is clearly visible only at later phases (after 450-500 days from the explosion, see Fig. 3).

A further spectrum was obtained about 13 months after the first one. Surprisingly there is no significant evidence for $\mathrm{H} \alpha$ or other H Balmer lines, while the emission feature at $7300 \AA$ is still quite prominent. This is indeed the only spectral line unequivocally visible in this spectrum.

In Fig. 5 three spectra of SN 1999ga are compared with those of the type IIL SN 1990K (Cappellaro et al. 1995b) and the type IIP SN 2004et (Sahu et al. 2006) ${ }^{2}$. The three SNe appear to have rather similar spectra at $\sim 120$ and $\sim 200$ days. The $\sim 120$ days spectra of all $\mathrm{SNe}$ show a prominent $\mathrm{H} \alpha$ line, although only in SN 1999ga $\mathrm{H} \alpha$ has a flat-topped profile. Subsequent spectra of the three $\mathrm{SNe}$ (phase of about 200 days) show a rather normal, rounded $\mathrm{H} \alpha$ profile which is expected in non-interacting SNe II. As a remarkable difference with the H-rich type IIP SN 2004et, very late spectra of SNe 1999ga and $1990 \mathrm{~K}$ obtained around 15 and 17.5 months (respectively)

\footnotetext{
2 The spectra of the comparison objects have been downloaded from the Online Supernova Spectrum Archive SUSPECT (http:// bruford.nhn. ou. edu/ suspect/index 1.html).
} 
Table 4. Pre- and late, post-explosion (ground-based and HST) images. The magnitudes of source $A$ in the ground-based observations are also reported in Col. 5.

\begin{tabular}{ccccccc}
\hline \hline Date & JD & Telescope & Filter & Exposure time (s) & Source A magnitude & Proposal ID / Source \\
\hline $1989-12-31$ & 2447891.53 & SSO 1 m & $\mathrm{H} \alpha$ & $3 \times 1000$ & & Ryder \& Dopita (1993) \\
$1989-12-31$ & 2447891.57 & SSO 1 m & $R_{\mathrm{c}}$ & $2 \times 500$ & $>18.76$ & Ryder \& Dopita (1993) \\
$1990-03-04$ & 2447955.45 & SSO 1 m & $V$ & $2 \times 250$ & $>20.70$ & Obs. S. Ryder \\
$1991-01-11$ & 2448267.62 & SSO 1 m & $I$ & $2 \times 250$ & $>20.20$ & Obs. S. Ryder \\
$1995-02-22$ & 2449771.06 & AAT & unfilt. & $300+60$ & $21.85 \pm 0.40$ & Obs. Whiteoak \& Koribalski \\
$1995-03-01$ & 2449777.57 & CTIO 1.5 m & $B$ & $2 \times 600$ & $22.76 \pm 0.32$ & Obs. G. Purcell \\
$1995-03-01$ & 2449777.58 & CTIO 1.5 m & $I$ & 300 & $21.37 \pm 0.37$ & Obs. G. Purcell \\
\hline $2006-01-28$ & 2453764.70 & ESO 2.2 m & $\mathrm{H} \alpha$ & $4 \times 720$ & & \\
$2006-01-29$ & 2453764.77 & ESO 2.2 m & $V$ & $2 \times 600$ & $22.07 \pm 0.09$ & 076.C-0888 (PI: Y. Bialetski) \\
$2006-01-30$ & 2453765.73 & ESO 2.2 m & $B$ & $2 \times 300$ & $22.89 \pm 0.08$ & 076.C-0888 (PI: Y. Bialetski) Bialetski) \\
$2006-11-29$ & 2454068.78 & ESO 2.2 m & $B$ & $4 \times 600$ & $22.87 \pm 0.22$ & MPI Time (PI: W. Hillebrandt) \\
\hline $2006-10-21$ & 54030.31 & HST & $F 435 W$ & 1580 & & 10803 (PI: S. Smartt) \\
$2006-10-21$ & 54030.37 & HST & $F 658 N$ & 1350 & & 10803 (PI: S. Smartt) \\
$2006-10-21$ & 54030.39 & HST & $F 814 W$ & 1200 & & 10803 (PI: S. Smartt) \\
\hline
\end{tabular}

* Magnitude from unfiltered image, rescaled to the $R$ band photometry.

SSO $1 \mathrm{~m}=1 \mathrm{~m}$-Telescope + CCD (Siding Spring Observatory, Australia); AAT = $4 \mathrm{~m}$ Anglo Australian Telescope + CCD (Siding Spring Observatory, Australia); CTIO $1.5 \mathrm{~m}=1.5 \mathrm{~m}$-Telescope + CCD (Cerro Tololo Inter-American Observatory, Chile); ESO $2.2 \mathrm{~m}=\mathrm{ESO} / \mathrm{MPI} 2.2 \mathrm{~m}$ Telescope + Wide Field Imager (La Silla, Chile); HST = Hubble Space Telescope + ACS/WFC Camera.

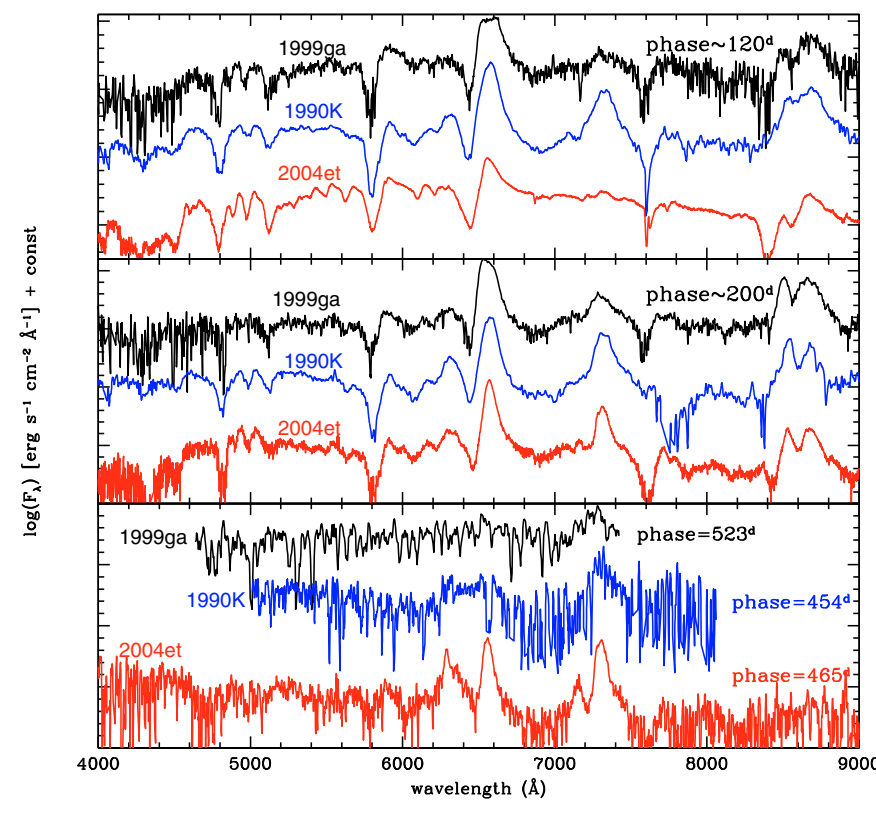

Fig. 5. Comparison between spectra of SN 1999ga, the type IIL SN 1990K (Cappellaro et al. 1995b) and the type IIP SN 2004et (Sahu et al. 2006) at different phases. The phases are computed with reference to an approximate estimate of the explosion dates. For SN 1990K we adopted $\mathrm{JD}=2448020$ as explosion time, which is about 17 days before the discovery epoch ( $\mathrm{JD}=2448037.3$ ). Our assumption is in good agreement with that of Cappellaro et al. (1995b), who estimated the explosion of SN 1990K to occur about two weeks before its discovery. Finally, for SN 2004et we adopt the same explosion epoch as Sahu et al. (2006) (JD = 2453270.5 , see also Li et al. 2005).

after their explosions surprisingly do not show any prominent $\mathrm{H} \alpha$ line (Fig. 5, bottom panel), but - this is more clearly visible in SN $1990 \mathrm{~K}$ - only a broad, weak bump around 6300-6400 A, which is mostly due to the [O I] $16300-6364$ doublet. The only (relatively) prominent feature is that at $\sim 7300 \mathrm{~A}$, possibly a blend of several forbidden lines ([CaII], [O II], [Fe II]). The lack of prominent $\mathrm{H} \alpha$ at very late times supports the scenario of a progenitor star with a small residual $\mathrm{H}$ envelope and, hence, a type IIL classification for SN 1999ga.
Apart from the peculiar $\mathrm{H} \alpha$ line profile in the earliest spectrum, there is little evidence that the $\mathrm{SN}$ is interacting with a CSM. The lack of the flattening in the optical light curves typical of SNe which are strongly interacting with a CSM is in good agreement with this scenario. As a further support, no emission was detected at the position of SN $1999 \mathrm{ga}$ in $6 \mathrm{~cm}(5170 \mathrm{MHz})$ images analysed by Harnett et al. (2004), neither in images obtained during the 1990s, before the SN explosion, or in a postexplosion image obtained on 2000 December 31 (i.e. more than one year after the explosion). Therefore, if the earliest optical spectrum may eventually suggest the presence of material lost by the progenitor in pre-SN mass loss events, the subsequent $\mathrm{SN}$ evolution allows us to exclude major ejecta-CSM interaction episodes.

\section{The birthplace of SN 1999ga}

A method to understand the nature of the star which exploded as SN 1999ga is to study the site of explosion, trying to derive information on the progenitor via the direct detection of the star (or constraining robust detection limits) in available pre-explosion images (e.g. Smartt et al. 2004; van Dyk et al. 2003; Maund \& Smartt 2005; Smartt et al. 2009). The site of explosion of SN 1999ga was occasionally monitored in the past using smallsize telescopes (see Table 4). A large sample of low-resolution archive images obtained using different filters and showing the explosion site over a period of about 17 years is presented in Fig. 6, while in Fig. 7 the region of the $\mathrm{SN}$ is shown in a sequence of $B$ band images obtained before, during and after the SN outburst, including a high-resolution HST image of the postexplosion site (panel D).

In particular, pre-explosion images were obtained during the 1990s at the $1 \mathrm{~m}$ Telescope and the $3.9 \mathrm{~m}$ Anglo Australian Telescope at Siding Spring Observatory (Australia), and the $1.5 \mathrm{~m}$ Telescope of the Cerro Tololo Inter-American Observatory (CTIO) in Chile (Fig. 6, inserts A to E, and Fig. 7, insert A). Some of the images here analysed were published in Ryder \& Dopita (1993). The best-quality pre-explosion images are those in the $B$ and $I$ bands obtained on March 1995 at the CTIO $1.5 \mathrm{~m}$-Telescope. Unfortunately, most pre-SN frames are not 


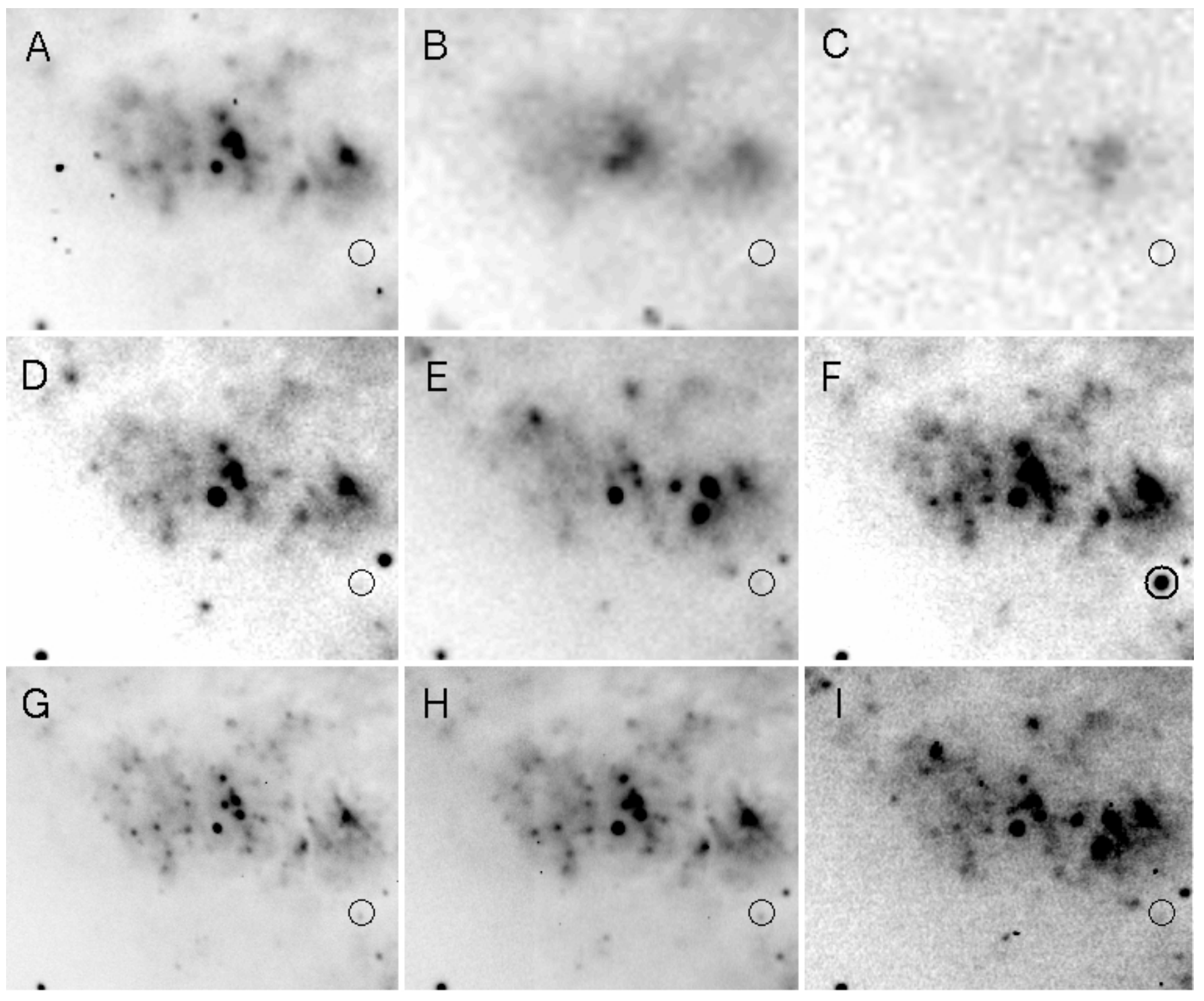

Fig. 6. The explosion site of SN 1999ga: low-resolution frames obtained with different ground-based telescopes. (A): pre-explosion $B$ band image obtained on March 1, 1995 with the $1.5 \mathrm{~m}$ CTIO telescope. (B) Pre-explosion $V$ band image obtained March 4, 1990 with the $1 \mathrm{~m}$ Telescope at Siding Spring Observatory (SSO). (C) Pre-explosion H $\alpha$ image obtained on January 31, 1989 at the SSO $1 \mathrm{~m}$ Telescope. (D) Pre-explosion I band image obtained on March 1, 1995 with the $1.5 \mathrm{~m}$ CTIO telescope. (E) Pre-explosion unfiltered image obtained on February 22,1995 at the Anglo Australian Telescope. (F) B band image of SN 1999ga obtained on December 29, 1999 with the ESO/MPI 2.2 m Telescope in La Silla. (G) Post-explosion $B$ band image obtained on January 30, 2006 with the ESO/MPI $2.2 \mathrm{~m}$ Telescope. (H) Post-explosion $V$ band image obtained on January 29, 2006 with the ESO/MPI $2.2 \mathrm{~m}$ Telescope. (I) Post-explosion H $\alpha$ image obtained on January 28, 2006 with the ESO/MPI 2.2 m Telescope. The SN location is marked by a circle. Only an extended source (denoted as source $A$ in the text) is visible both in the pre- and post-explosion images in the SN vicinity. In all figures, north is up, east is to the left.

deep enough to provide robust detection limits for the putative progenitor star.

One problem is the SN location, which is on the northern edge of an elongated luminous region (that will be labelled hereafter as source A, see Figs. 6 and 7), visible in most pre- and post-explosion images. This region is particularly luminous in the $B$ band, indicating that it is probably associated with luminous blue stars. Interestingly, source $A$ is faint in the $\mathrm{H} \alpha$ images. This, together with the lack of evidence in the SN spectra of narrow lines from the galactic background, suggests a stellar nature (possibly stellar clusters) for this vast source near the $\mathrm{SN}$ location, rather than a star-forming region. Since the SN exploded at the edge of source $A$, it is very difficult to extrapolate the flux contribution of a single star from that of the entire environment. We measured the integrated magnitudes of the unresolved source $A$ in the ground-based images before and several years after the SN explosion, and we found (within the errors) no significant differences. This implies that the contribution of the progenitor of SN 1999ga to the flux of the whole extended region was negligible. The magnitudes of source $A$ as derived in the low resolution images are reported in Table 4 (Col. 6).

We also collected some deep post explosion images (Fig. 6, inserts G, H, I; Fig. 7, insert C) obtained with the ESO/MPI $2.2 \mathrm{~m}$ Telescope in January (under the proposal ID: 076.C-0888, PI: Y. Bialetski) and November 2006 (reserved MPI time, program ID 078.A-9046(A), PI: W. Hillebrandt). We used the best seeing $B$-band images (those of January 2006) as templates in our attempt to recover the progenitor star in the March 1995 $B$-band image. After combining the best seeing images obtained at the same epoch, and after geometrical and photometric 

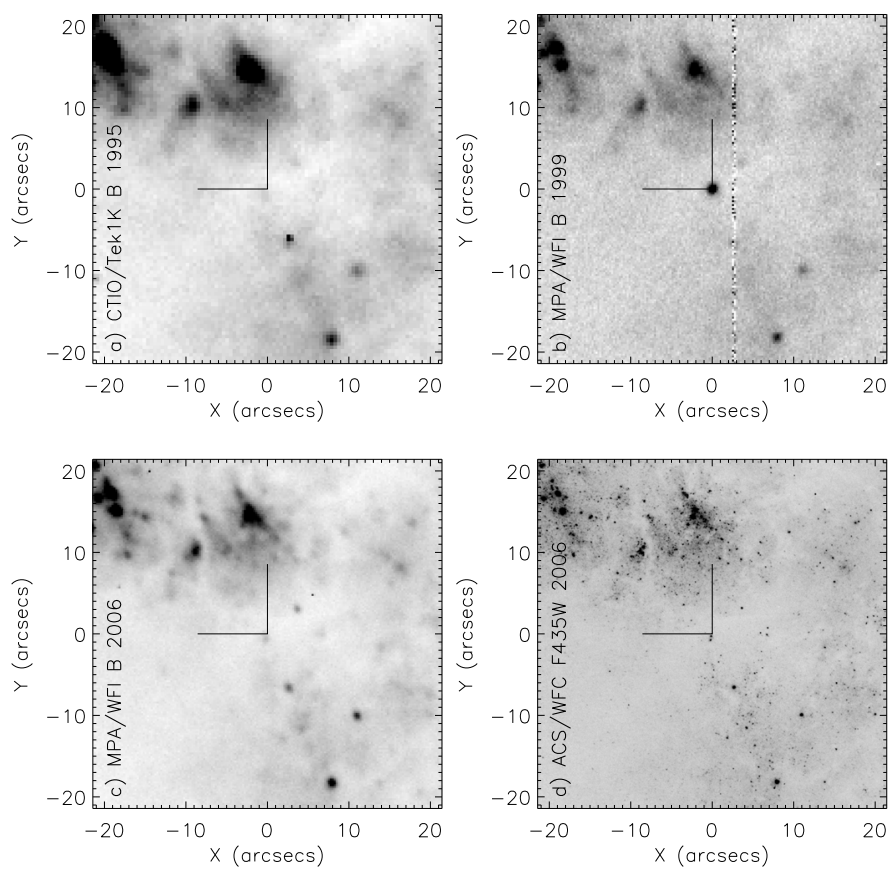

Fig. 7. The explosion site of SN 1999ga in the B band. Top-left: low resolution, pre-explosion CTIO 1.5-m telescope image obtained on March 1, 1995. Top-right: low-resolution SN image obtained on December 29, 1999 with the 1.54-m Danish Telescope (La Silla, Chile) equipped with DFOSC. Bottom-left: late time low resolution frame obtained on January 30, 2006 with the $2.2 \mathrm{~m}$ ESO/MPI Telescope (plus Wide Field Camera) in La Silla. Bottom-right: late time (October 21 , 2006) ACS HST image (filter $F 435 W$ ) of the explosion region. Information on these images is reported in Table 4. All images are centered at the SN position and oriented such that north is up and east is to the left.

registration of the pre- and post-explosion images, we subtracted the latter image from the former. With this procedure, the whole host galaxy and, hence, also the emitting region in the SN vicinity were removed. Nevertheless, any attempt to recover the progenitor star in the $B$-band failed, since there was no evidence of the progenitor of SN 1999ga in the subtracted image at the SN position at a $B$-band magnitude brighter than 23.15. Similar attempts were made with other images, and none showed evidence of the presence of any star at the position of the SN. Adopting the distance and reddening estimates discussed in Sect. 2, we obtain $M_{B} \gtrsim-9.3$ which is not, obviously, a very significant detection limit.

In order to study in more detail the structure of the extended source A, the site was targeted by the Hubble Space Telescope (HST) on October 21st, 2006, 7 years after the SN explosion (proposal ID: 10803, PI: S. J. Smartt). Source A, which appears to be elongated (roughly) in the North-South direction in the ground based images, in reality consists of two major sources (plus a few much fainter sources visible in their vicinity), as one can clearly gather from the high-resolution HST images (see blow-up panels of the F814W HST/ACS image, Fig. 8). Both these sources are extended with FWHM which is about twice that of the stellar PSF, so that we suggest that they are both compact stellar clusters.

We performed PSF-fitting photometry using the DOLPHOT package. DOLPHOT classifies the southern source as a single,

${ }^{3}$ DOLPHOT is a stellar photometry package that was adapted from HSTphot (Dolphin 2000) for general use. point-like object (type 1) and the northern source as extended (type 5). However, rather significant residuals are visible at the sites of both objects after PSF subtraction, and we conclude that no one of them is well fit by this photometry package.

Aperture photometry of these objects is complicated by the non-stellar nature of their PSFs. This makes it difficult to define aperture corrections and the objects are too close one another to use a large aperture.

The BAOLAB task ISHAPE (Larsen 1999, 2004) was used to determine the intrinsic size and shape of these assumed clusters. ISHAPE convolves an analytical function that is assumed to represent the actual source (e.g. a delta function for a star) with the stellar PSF. ISHAPE then compares this convolved function to the data to determine the best fit and therefore the intrinsic FWHM of the source function. An elliptical Moffat function with a power index of 1.5 was used to fit our proposed clusters. The resulting parameters for the southern and northern sources (computed for all HST images) are reported in Table 5.

It is worth noting that the intrinsic size, major/minor axis ratio and position angle of each object are consistent between the two broadband filters, while in $\mathrm{H} \alpha(F 658 N)$ they appear to have completely different shapes and orientations. In the case of the southern source, its extent in the $\mathrm{H} \alpha$ image is significantly larger than those derived in the broadband images. These measurements suggest that there is nebular $\mathrm{H} \alpha$ emission which is significantly elongated relative to the the cluster.

The BAOLAB task MKCMPPSF was used to calculate synthetic PSF matching of these two sources in each filter. These were added to fake images and used to calculate appropriate aperture corrections for the objects. This resulted in the corrected aperture photometry reported in Table 5 (bottom).

With the distance and extinction toward SN 1999ga adopted in this paper (see Sect. 2), we obtain absolute magnitudes of $F 435 W \sim-8.9$ and $F 814 W \sim-9.5$ for both objects ${ }^{4}$, which are certainly brighter than we would expect single stars to be.

Finally, the half-light/effective radii of the clusters from the $\mathrm{F} 814 \mathrm{~W}$ image was computed and found to be $R(\mathrm{eff})_{\text {Northern }}=$ $9.5 \pm 0.4 \mathrm{pc}$ and $R(\mathrm{eff})_{\text {Southern }}=5.5 \pm 0.3 \mathrm{pc}$. The northern cluster has a projected separation from the $\mathrm{SN}$ position of about $28 \pm$ 5 pc (7.3 \pm 1.3 pixels). Although previous studies allowed constraints to be placed on SN progenitors by estimating the ages and the main sequence turn-off masses of host stellar clusters (Maíz-Apellániz et al. 2004; Crockett et al. 2008a,b; Östlin et al. 2008), in the case of SN 1999ga the progenitor was probably too far from the northern cluster to claim that it was coeval. Therefore, despite the large amount of pre- and post-explosion images available for NGC 2442, no robust information can be derived for the progenitor star of SN 1999ga, since the only thing we can exclude is that the precursor was a very luminous star (e.g. a luminous blue variable).

\section{Discussion and conclusions}

Despite lacking early-time photometric monitoring of SN 1999ga, which would have allowed us to conclusively discriminate between the different subtypes of SNe II, we believe that spectroscopy has provided enough evidence to support a designation of type IIL for this object. The non-detection of $\mathrm{H} \alpha$ in the latest spectrum, in particular, indicates that the

\footnotetext{
4 Note that the adopted extinction is not necessarily appropriate for these proposed clusters. However, without photometry in more filters and/or spectra of these objects, it is not possible to provide a direct measurement of the extinction towards the two sources.
} 

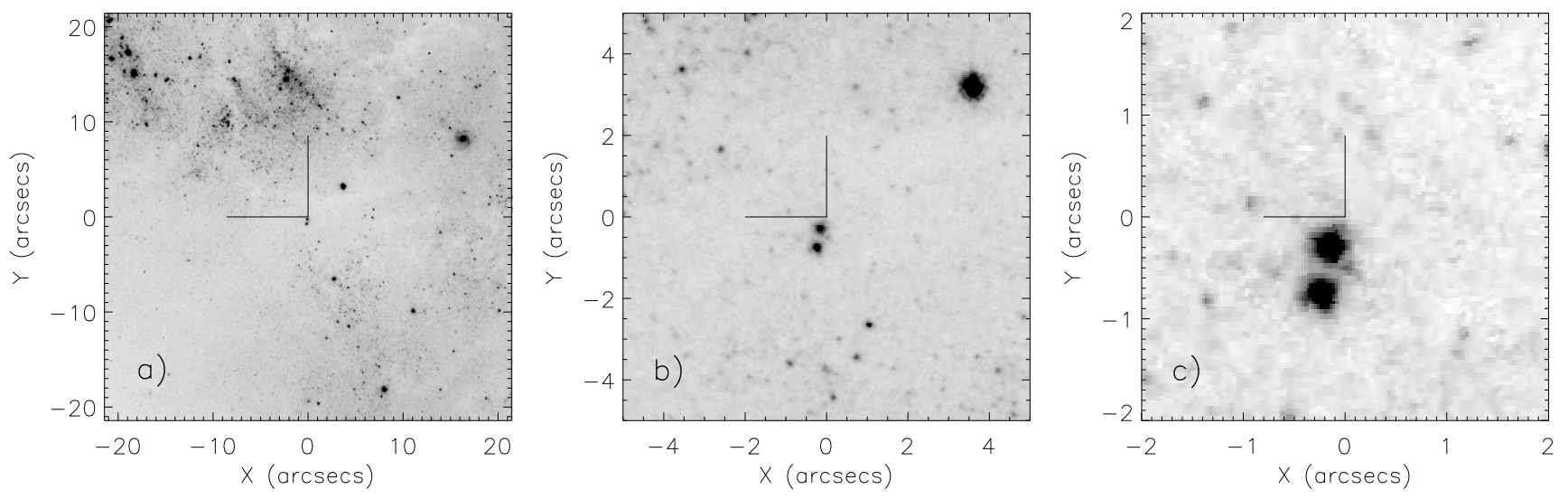

Fig. 8. HST $F 814 W$ band (roughly I) image obtained 7 years after the SN explosion (left panel) and blow ups of the SN region: $\times 4$ (central panel) and $\times 10$ (right panel). The SN position is marked by the cross-hairs, and no source is visible at that position. The high resolution of the HST/ACS images allows us to resolve source $A$ into two main components (probably two stellar clusters) plus other (though much fainter) sources. The images are centered at the SN position. North is up, east to the left.

Table 5. Basic parameters for the two putative clusters forming Source $A$, as derived by BAOLAB/ISHAPE in each of the three HST filters.

\begin{tabular}{cccc}
\hline \hline Source & $F 435 W$ & $F 658 N$ & $F 814 W$ \\
\hline \multicolumn{5}{c}{ FWHM of semi-major axis (pixels) } \\
Northern & 1.94 & 1.96 & 2.22 \\
Southern & 1.16 & 1.70 & 1.37 \\
\hline \multicolumn{5}{c}{ Ratio of major/minor axis } \\
Northern & 0.98 & 0.61 & 0.95 \\
Southern & 0.72 & 0.44 & 0.83 \\
\hline \multicolumn{5}{c}{ Position Angle (degrees) } \\
Northern & -43.9 & -2.6 & -48.1 \\
Southern & -14.1 & -69.0 & -14.6 \\
\hline \multicolumn{5}{c}{ Photometry (Vegamag) } \\
Northern & 23.49 & 22.35 & 21.95 \\
Southern & 23.62 & 22.46 & 22.09 \\
\hline
\end{tabular}

residual $\mathrm{H}$ envelope of the exploding star was not very massive (although probably more massive than that of a type IIb SN).

A sub-division of SNe IIL into two groups (bright, like SN 1979C, and regular events, depending on their intrinsic luminosities) was proposed by a number of authors (Young \& Branch 1989; Gaskell 1992; Patat et al. 1994). Richardson et al. (2002) estimated the average absolute magnitudes for bright and normal SNe IIL to be around $M_{B} \approx-19.3$ and $M_{B} \approx-17.6$, respectively. It is evident from a simple check of the absolute magnitudes of SN 1999ga at any time that this object is much fainter than regular SNe IIL. This may possibly indicate the existence of a lowluminosity tail in the luminosity distribution of type IIL SNe, similar to that already observed in SNe IIP (Hamuy 2003; Pastorello et al. 2004). Even if the peak luminosity can be questioned because of the late discovery of SN 1999ga, the radioactive tail is much fainter than that observed in other SNe IIL (see Fig. 3) and is comparable in luminosity with those of lowluminosity SNe IIP (Pastorello et al. 2004). This faint radioactive tail is consistent with the ejection of a small mass of ${ }^{56} \mathrm{Ni}$ $\left(\sim 10^{-2} M_{\odot}\right)$, an amount which is only marginally higher than that reported for low-luminosity SNe IIP (Pastorello et al. 2004, 2009). However, in stark contrast to faint SNe IIP, the broad spectral features observed in the SN 1999ga spectra are indicative of high-velocity ejecta (5000-6000 $\mathrm{km} \mathrm{s}^{-1}$, see Sect. 4). These two observed quantities suggest a normal explosion energy and moderate mass of the ejected material (including radioactive ${ }^{56} \mathrm{Ni}$ ) for $\mathrm{SN} 1999 \mathrm{ga}$. There is indeed robust evidence from spectroscopy that the mass of the $\mathrm{H}$ envelope was rather small and/or that there was a non-negligible amount of CSM, resulting from mass loss episodes during the late stages of the stellar life. Several attempts have been made to estimate the explosion parameters of past SNe IIL. The observed evolution of the nebular $\mathrm{H} \alpha$ line in the cases of the bright SNe 1980K and 1990K (Cappellaro et al. 1995b, and references therein) was well reproduced by the models of Chugai (1991) with $5 M_{\odot}$ of ejecta, canonical explosion energy $\left(E_{0}=10^{51} \mathrm{erg}\right)$ and intermediate ${ }^{56} \mathrm{Ni}$ mass $\left(\mathrm{M}\left({ }^{56} \mathrm{Ni}\right)=0.075 \mathrm{M}_{\odot}\right)$. Slightly higher values of the above parameters are probably necessary to account for the higher luminosity of $\mathrm{H} \alpha$ in SN 1979C, even though the luminous light curve peak probably did not result from anomalous explosion parameters, but through reprocessing of UV light in a shell generated by pre-SN wind (Bartunov \& Blinnikov 1992).

In Fig. 9 we compare the luminosity evolution of the $\mathrm{H} \alpha$ line in SN 1999ga with models of $\mathrm{H} \alpha$ luminosity expected in type II SNe, assuming that the source of the luminosity is purely radioactive decay (Chugai 1990, 1991). The models were obtained adopting ejected masses of $20 M_{\odot}$ (blue dashed line), $14 M_{\odot}$ (green long-dashed line), $5 M_{\odot}$ (dotted red line), and computed with $E_{0}=10^{51} \mathrm{erg}$ and $M\left({ }^{56} \mathrm{Ni}\right)=0.075 M_{\odot}$. In Fig. 9 the luminosity evolution of other typical nebular lines ([O I] $\lambda \lambda 6300-6364$, the [Ca II] plus [O II] blend around $7300 \AA$ and the Ca II NIR triplet) is also shown. We note that the $\mathrm{H} \alpha$ luminosities are systematically lower than those predicted by Chugai's models with $0.075 M_{\odot}$ of ${ }^{56} \mathrm{Ni}$, although the early decline rate is quite consistent with that of the $14 M_{\odot}$ model. We therefore rescaled the $14 M_{\odot}$ model of Chugai (1990) to $M\left({ }^{56} \mathrm{Ni}\right)=0.015 M_{\odot}$. This value is consistent with the ${ }^{56} \mathrm{Ni}$ mass deduced from the late time light curve of SN 1999ga (see Sect. 3). The rescaled $14 M_{\odot}$ model is shown in Fig. 9 (solid green line), and fits reasonably well to the observed $\mathrm{H} \alpha$ luminosities of SN 1999ga only at earlier epochs, while it fails to match the late-time $\mathrm{H} \alpha$ detection limit. A lower ejected mass would help to better reproduce the faster $\mathrm{H} \alpha$ luminosity decline observed at late time, although it would fail to fit the earliest observed point of SN 1999ga. A reasonable range for the total ejected mass of SN $1999 \mathrm{ga}$ is therefore $6-8 M_{\odot}$, with only $1-2_{\odot}$ of $\mathrm{H}$.

SNe IIL belong to a sequence of supernova types produced by progenitors with increasing mass loss occurring during the 


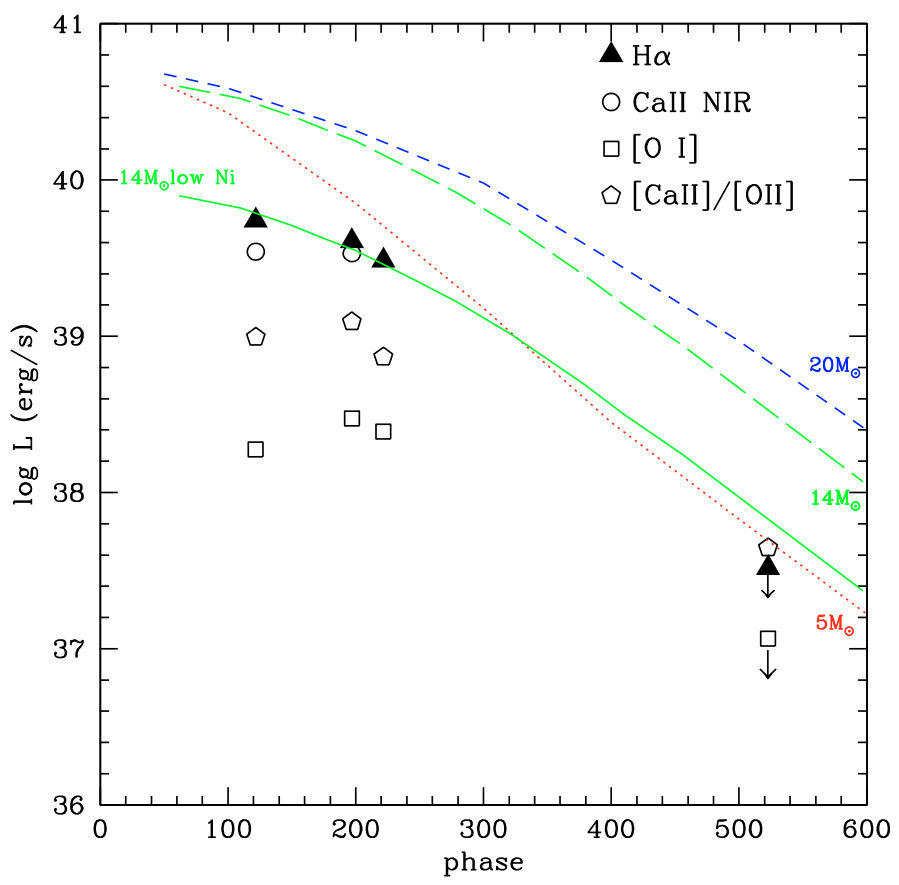

Fig. 9. Luminosity evolution of the main nebular features in the spectra of SN 1999ga. Models of Chugai (1991) showing the evolution of $\mathrm{H} \alpha$ in $\mathrm{SNe}$ II under the assumption of pure radioactive decay of ${ }^{56} \mathrm{Co}$ are also shown. The dotted red line marks the $\mathrm{H} \alpha$ luminosity evolution for an ejected mass of $5 M_{\odot}$, the long-dashed green line for $14 M_{\odot}$ and the dashed blue line for $20 M_{\odot}$. All models were computed with an explosion energy of $10^{51}$ erg and an ejected ${ }^{56} \mathrm{Ni}$ mass of $0.075 M_{\odot}$. The solid green lines is the $14 M_{\odot}$ model, but rescaled to $0.015 M_{\odot}$ of ${ }^{56} \mathrm{Ni}$.

late phases of their evolution, i.e. SN IIP $\rightarrow$ SN IIL $\rightarrow$ SN IIb $\rightarrow$ $\mathrm{SN} \mathrm{Ib} \rightarrow$ SN Ic (e.g. Nomoto 1997; Chevalier 2006). However, the nature of their progenitor stars is still unclear. Two channels have been proposed for type IIL SNe: single, massive progenitors $\left(M_{\text {ZAMS }} \geq 20 M_{\odot}\right)$, and lower mass stars $\left(7-10 M_{\odot}\right.$, Swartz et al. 1991) in binary systems, where the mass loss is triggered by the companion star.

Unfortunately, the lack of high-resolution, deep, preexplosion HST images prevents us from providing robust constraints on the nature of the progenitor of SN 1999ga. With the remarkable exception of the detections of the K supergiant progenitor of SN 1993J (Aldering et al. 1994; Maund et al. 2004; Maund \& Smartt 2009) and the luminous hypergiant progenitor of SN 2005gl (Gal-Yam et al. 2007; Gal-Yam \& Leonard 2009), most attempts to identify the progenitors of stripped (or partially stripped) envelope core-collapse $\mathrm{SNe}$ have failed (Smartt et al. 2002; van Dyk et al. 2003; Maund \& Smartt 2005; Maund et al. 2005; Gal-Yam et al. 2005; Crockett et al. 2007; Crockett et al. 2008a) or are disputed (Crockett et al. 2008b). However, the lack of any detection in ground based pre-explosion images, the analysis of the surrounding environment, and the observed $\mathrm{SN}$ properties (unusual spectral and $\mathrm{H} \alpha$ luminosity evolutions, sub-luminous light curves, small ${ }^{56} \mathrm{Ni}$ mass) all suggest that the precursor of SN 1999ga at the time of core-collapse was likely a moderate-mass star $\left(8-10 M_{\odot}\right)$, with a moderate-to-low mass residual $\mathrm{H}$ envelope. The fact that the $\mathrm{H}$ lines and not the He lines dominate the SN spectra in the early nebular phase is indicative that the star retained a significant mass $\left(1-2 M_{\odot}\right)$ of $\mathrm{H}$ at the time of the explosion. This is more than the few $\times 10^{-1} M_{\odot}$ expected for type IIb SNe (e.g. SN 2008ax, see Pastorello et al. 2008 b, and references therein), but surely less than the several solar masses estimated for a normal type IIP SN (e.g. Nadyozhin 2003). Although the ejecta mass implies a moderate mass star $\left(8-10 M_{\odot}\right)$ at explosion, the initial mass is somewhat uncertain. As the hydrogen mass is low, the progenitor likely lost mass through either stellar winds or mass-transfer to a binary. In either case, the amount of mass-loss could be significant (e.g. 5-10 $M_{\odot}$ ). The pre-explosion images are not deep enough to distinguish between these two scenarios and the SN is not close enough to the compact clusters to assume that it is coeval with their stellar population.

SN 1999ga is probably the first (relatively) under-luminous, ${ }^{56} \mathrm{Ni}$ poor core-collapse supernova that can be classified as type IIL. The velocity of the ejecta, as constrained by the width of the spectral lines, is not as low as observed in faint type II-P SNe (Pastorello et al. 2004). This provides further evidence, in contrast with SNe IIP, for a moderate mass ejected by SN 1999ga. The detection of this kind of objects is a rare event, probably because of their faint nature coupled with a fast photometric evolution. The discovery of SN 1999ga should be seen as motivation for searches for more extreme, fast-evolving, sub-luminous, envelope-stripped core-collapse SNe. These are expected to occur (e.g. Woosley 1993) but, apart from a few remarkable exceptions (see Valenti et al. 2009), usually elude detection. Similar SNe have been also proposed to be responsible for a class of long $\gamma$-ray bursts that do not show evidence of associated SNe (Della Valle et al. 2006; Fynbo et al. 2006; Gal-Yam et al. 2006; Gehrels et al. 2006; Ofek et al. 2007; Dado et al. 2008).

Acknowledgements. We ackwnoledge the anonymous referee for useful comments on the manuscript. This paper is based on observations made with the NASA/ESA Hubble Space Telescope, with the Perth-Lowell $0.61 \mathrm{~m}$ Cassegrain Telescope of the Perth Observatory (Australia), and at the $1.54 \mathrm{~m}$ Danish Telescope, the ESO/MPI $2.2 \mathrm{~m}$ Telescope and the $3.6 \mathrm{~m}$ Telescope at the European Southern Observatory (La Silla, Chile) under program IDs 65.H-0292 (PI: M. Turatto) 66.D-0683 (PI: M. Turatto). Support for program GO-10803 was provided by NASA through a grant from the Space Telescope Science Institute, which is operated by the Association of Universities for Research in Astronomy, Inc., under NASA contract NAS 5-26555. This paper is also partly based on observations made with the European Southern Observatory telescopes obtained from the ESO/ST-ECF Science Archive Facility. We also made use of data obtained from the Anglo Australian Telescope Archive which is maintained as part of the CASU Astronomical Data Centre at the Institute of Astronomy, Cambridge. S.B., E.C. and M.T. are supported by the Italian Ministry of Education via the PRIN 2006 No. 2006022731-002. S.M. acknowledges funding from the Academy of Finland (project 8120503).

\section{References}

Aldering, G., Humphreys, R. M., \& Richmond, M. 1994, AJ, 107, 662

Aschenbach, B. 2007, Supernova 1987A: 20 Years after - Supernovae and Gamma-Ray Bursters, AIP Conf. Proc., 937, 33

Bajaja, E., Wielebinski, R., Reuter, H.-P., Harnett, J. I., \& Hummel, E. 1995, A\&AS, 114, 147

Bajaja, E., Agüero, E., \& Paolantonio, S. 1999, A\&AS, 136, 177

Balinskaya, I. S., Bychkov, K. V., \& Neisvestny, I. S. 1980, A\&A, 85, L19

Barbon, R., Ciatti, F., \& Rosino, L. 1982a, A\&A, 166, 35

Barbon, R., Ciatti, F., Rosino, L., Ortolani, S., \& Rafanelli, P. 1982b, A\&A, 166, 43

Bartel, N., \& Bietenholz, M. F. 2003, ApJ, 591, 301

Bartunov, O. S., \& Blinnikov, S. I. 1992, SvAL, 18, 43

Bekki, K., Koribalski, B. S., Ryder, S. D., \& Couch, W. J. 2005, MNRAS, 357, L21

Benetti, S. 2000, Mem. Soc. Astron. Ital., 71, 323

Benetti, S., \& Neuhauser, R. 1996, IAU Circ., 643

Benetti, S., Cappellaro, E., Danziger, I. J., et al. 1998, MNRAS, 294, 448

Benetti, S., Turatto, M., Cappellaro, E., Danziger, I. J., \& Mazzali, P. A. 1999,

MNRAS, 305, 811

Branch, D., Falk, S. W., Uomoto, A. K., et al. 1981, ApJ, 244, 780 
Buta, R. J. 1982, PASP, 94, 578

Cappellaro, E., della Valle, M., Iijima, T., \& Turatto, M. 1990, A\&A, 228, 61 Cappellaro, E., Danziger, I. J., \& Turatto, M. 1995a, MNRAS, 277, 106

Cappellaro, E., Danziger, I. J., Della Valle, M., Gouiffes, C., \& Turatto, M. 1995b, A\&A, 293, 723

Cappellaro, E., Benetti, S., Turatto, M., \& Pastorello, A. 2000, IAU Circ., 7380

Chevalier, R. A. 2006, Proceedings of 2006 STScI May Symposium on Massive Stars [arXiv: astro-ph/0607422]

Chugai, N. N. 1990, SvAL, 16, 457

Chugai, N. N. 1991, MNRAS, 250, 513

Chugai, N. N., Chevalier, R. A., \& Utrobin, V. P. 2007, ApJ, 662, 1136

Clocchiatti, A., Benetti, S., Wheeler, J. C., et al. 1996, AJ, 111, 1286

Crockett, R. M., Smartt, S. J., Eldridge, J. J., et al. 2007, MNRAS, 381, 835

Crockett, R. M., Maund, J. R., Smartt, S. J., et al. 2008a, ApJ, 672, L99

Crockett, R. M., Eldridge, J. J., Smartt, S. J., et al. 2008b, MNRAS, 391, L5

Dado, S., Dar, A., De Rújula, A., \& Plaga, R. 2008, ApJ, 678, 353

Dessart, L., \& Hillier, D. J. 2008, MNRAS, 383, 57

Della Valle, M., Chincarini, G., Panagia, N., et al. 2006, Nature, 444, 1050

Di Carlo, E., Massi, F., Valentini, G., et al. 2002, ApJ, 573, 144

Dolphin, A. E. 2000, PASP, 112, 1383

Elmegreen, D. M., Sundin, M., Sundelius, B., \& Elmegreen, B. G. 1991, A\&A, 244,52

Elmhamdi, A., Danziger, I. J., Chugai, N., et al. 2003, MNRAS, 338, 939

Fassia, A., Meikle, W. P. S., Vacca, W. D., et al. 2000, MNRAS, 318, 1093

Fesen, R. A., \& Matonick, D. M. 1993, ApJ, 407, 110

Fesen, R. A., Gerardy, C. L., Filippenko, A. V., et al. 1999, AJ, 117, 725

Foley, R. J., Smith, N., Ganeshalingam, M., et al. 2007, ApJ, 657, L105

Fynbo, J. P. U., Watson, D., Thöne, C. C., et al. 2006, Nature, 444, 1047

Gal-Yam, A., \& Leonard, D. C. 2009, Nature, 458, 865

Gal-Yam, A., Fox, D. B., Kulkarni, S. R., et al. 2005, ApJ, 630, L29

Gal-Yam, A., Fox, D. B., Price, P. A., et al. 2006, Nature, 444, 1053

Gal-Yam, A., Leonard, D. C., Fox, D. B., et al. 2007, ApJ, 656, 372

Gaskell, C. 1992, ApJ, 389, L17

Gehrels, N., Norris, J. P., Barthelmy, S. D., et al. 2006, Nature, 444, 1044

Gezari, S., Halpern, J. P., Grupe, D., et al. 2009, ApJ, 690, 1313

Hamuy, M. 2003, ApJ, 582, 905

Harnett, J., Ehle, M., Fletcher, A., et al. 2004, A\&A, 421, 571

Houghton, S. 1998, Ph.D. Thesis, Univ. New South Wales

Immler, S., Pietsch, W., \& Aschenbach, B. 1998, A\&A, 331, 601

Immler, S., Fesen, R. A., Van Dyk, S. D., et al. 2005, ApJ, 632, 283

Jeffery, D., \& Branch, D. 1990, in Supernovae, ed. J. C. Wheeler, T. Piran, \& S.

Weinberg (Singapore: World Scientific Publishing Co.), 149

Jha, S., Challis, P., Kirshner, R., \& Berlind, P. 2000, IAU Circ., 7381

Landolt, A. U. 1992, AJ, 104, 372L

Larsen, S. S. 1999, A\&AS, 139, 393

Larsen, S. S. 2004, A\&A, 416, 537

Leibundgut, B. Kirshner, R. P., Pinto, P. A., et al. 1991, ApJ, 372, 531

Li, W., Van Dyk, S. D., Filippenko, A. V., \& Cuillandre, J.-C. 2005, PASP, 117, 121

Liu, Q.-Z., Hu, J.-Y., Hang, H.-R., et al. 2000, A\&AS, 144, 219

Maíz-Apellániz, J., Bond, H. E., Siegel, M. H., et al. 2004, ApJ, 615, L113

Marcaide, J. M., Pérez-Torres, M. A., Ros, E., et al. 2002, A\&A, 384, 408

Misra, K., Pooley, D., Chandra, P., et al. 2007, MNRAS, 381, 280

Maund, J. R., \& Smartt, S. J. 2005, MNRAS, 360, 288

Maund, J. R., \& Smartt, S. J. 2009, Science, 324, 486

Maund, J. R., Smartt, S. J., Kudritzki, R. P., Podsiadlowski, P., \& Gilmore, G. F. 2004, Nature, 427, 129

Maund, J. R., Smartt, S. J., \& Schweizer, F. 2005, ApJ, 630, L33

Mihos, J. C., \& Bothun, G. D. 1997, ApJ, 481, 741

Miller, A. A., Chornock, R., Perley, D. A., et al. 2009, ApJ, 690, 1303

Montes, M. J., Weiler, K. W., Van Dyk, S. D., et al. 2000, ApJ, 532, 1124

Nadyozhin, D. K. 2003, MNRAS, 346, 97
Nomoto, K., Iwamoto, K., Suzuki, T., et al. 1997, Compact stars in binaries, held 15 through 19 August 1994, as part of the 22nd General Assembly of the IAU in The Hague, The Netherlands, ed. J. van Paradijs, E. P. J. van den Heuvel, \& E. Kuulkers (Dordrecht: Kluwer Academic Publishers), Proc. IAU Symp., 165,119

Ofek, E. O., Cenko, S. B., Gal-Yam, A., et al. 2007, ApJ, 662, 1129

Östlin, G., Zackrisson, E., Sollerman, J., Mattila, S., \& Hayes, M. 2008, MNRAS, 387, 1227

Panagia, N., Vettolani, G., Boksenberg, A., et al. 1980, MNRAS, 192, 861

Pastorello, A., Zampieri, L., Turatto, M., et al. 2004, MNRAS, 347, 74

Pastorello, A., Baron, E., Branch, D., et al. 2005, MNRAS, 360, 950

Pastorello, A., Sauer, D., Taubenberger, S., et al. 2006, MNRAS, 370, 1752

Pastorello, A., Smartt, S. J., Mattila, S., et al. 2007, Nature, 447, 829

Pastorello, A., Mattila, S., Zampieri, L., et al. 2008a, MNRAS, 389, 113

Pastorello, A., Kasliwal, M. M., Crockett, R. M., et al. 2008b, MNRAS, 389, 955

Pastorello, A., Valenti, S., Zampieri, L., et al. 2009, MNRAS, 394, 2266

Patat, F., Barbon, R., Cappellaro, E., \& Turatto, M. 1993, A\&AS, 98, 443

Patat, F., Barbon, R., Cappellaro, E., \& Turatto, M. 1994, A\&A, 282, 731

Quimby, R. M., Aldering, G., Wheeler, J. C., et al. 2007, ApJ, 668, L99

Ray, A., Petre, R., \& Schlegel, E. M. 2001, AJ, 122, 966

Richardson, D., Branch, D., Casebeer, D., et al. 2002, AJ, 123, 745

Rubenstein, E. P. 1999, IAU Circ., 7319

Ryder, S. D., \& Dopita, M. A. 1993, ApJS, 88, 415

Ryder, S. D., Koribalski, B., Staveley-Smith, L., et al. 2001, ApJ, 555, 232

Sahu, D. K., Anupama, G. C., Srividya, S., \& Muneer, S. 2006, MNRAS, 372, 1315

Salvo, M., Rizzi, L., Pastorello, A., \& Turatto, M. 1999, IAU Circ., 7340

Sandage, A., \& Bedke, J. 1994, The Carnegie Atlas of Galaxies

Schlegel, E. M. 1990, MNRAS, 244, 269

Schlegel, E. M. 1995, Rep. Prog. Phys., 58, 1375

Schlegel, D. J., Finkbeiner, D. P., \& Davis, M. 1998, ApJ, 500, 525

Smartt, S. J., Gilmore, G. F., Tout, C. A., \& Hodgkin, S. T. 2002, ApJ, 565, 1089

Smartt, S. J., Maund, J. R., Hendry, M. A., et al. 2004, Science, 303, 499

Smartt, S. J., Eldridge, J. J., Crockett, R. M., \& Maund, J. R. 2009, MNRAS, 395,1409

Swartz, D. A., Wheeler, J. C., \& Harkness, R. P. 1991, ApJ, 374, 266

Tsvetkov, D. Y. 1983, Perem. Zvezdy, 22, 39

Tsvetkov, D. Yu., Volnova, A. A., Shulga, A. P., et al. 2006, A\&A, 460, 769

Turatto, M., Cappellaro, E., Danziger, I. J., et al. 1993a, MNRAS, 262, 128

Turatto, M., Cappellaro, E., Benetti, S., \& Danziger, I. J. 1993b, MNRAS, 265, 471

Turatto, M., Benetti, S., \& Cappellaro, E. 2003, From Twilight to Highlight: The Physics of Supernovae: Proceedings of the ESO/MPA/MPE Workshop Held at Garching, Germany, 29-31 July 2002, ed. W. Hillebrandt, \& B. Leibundgut (Springer-Verlag), 200

Uomoto, A., \& Kirshner, R. P. 1986, ApJ, 308, 685

Valenti, S., et al. 2009, Nature, in press [arXiv:0901.2074]

van Dyk, S. D., Weiler, K. W., Sramek, R. A., et al. 1996, AJ, 111, 1271

van Dyk, S. D., Peng, C. Y., Barth, A. J., et al. 1999, PASP, 111, 313

van Dyk, S. D., Li, W., \& Filippenko, A. V. 2003, PASP, 115, 21

de Vaucouleurs, G., de Vaucouleurs, A., Buta, R., Ables, H. D., \& Hewitt, A. V. 1981, PASP, 93, 36

Weiler, K. W., van Dyk, S. D., Discenna, J. L., Panagia, N., \& Sramek, R. A. 1991, ApJ, 380, 161

Whitelock, P. A., et al. 1989, MNRAS, 230, 7

Woodings, S. J., Williams, A. J., Martin, R., Burman, R. R., \& Blair, D. G. 1998, MNRAS, 301, L5

Woodings, S., Martin, R., Williams, A., Biggs, J., \& Verveer, A. 1999, IAU Circ., 7316

Woosley, S. E. 1993, ApJ, 405, 273

Young, T., \& Branch, D. 1989, ApJ, 342, L79 